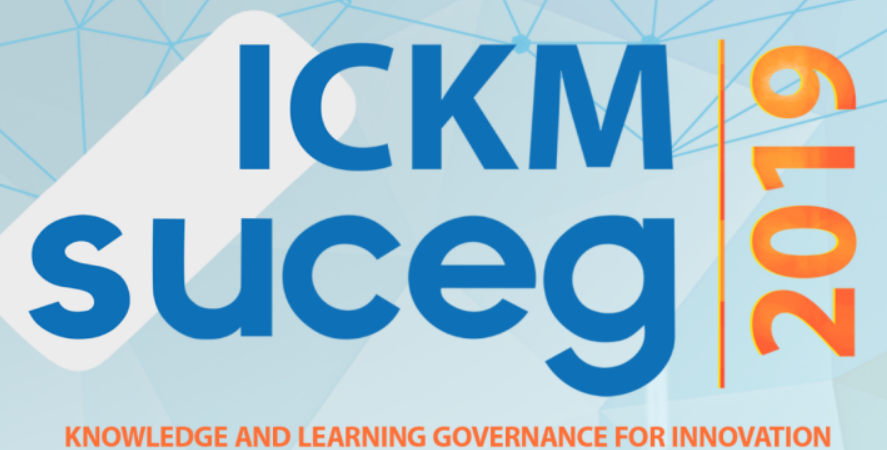

\title{
ANÁLISE DA RELAÇÃO ENTRE LIDERANÇA E CAPACIDADE ABSORTIVA DO CONHECIMENTO: uma revisão sistemática da literatura
}

\section{Daniela de Oliveira Massad}

Doutoranda do Programa de Pós-Graduação em Engenharia e Gestão do Conhecimento, UFSC, 0000-0003-1095-8856,danimassad@gmail.com

\section{Daniele Santos de Oliveira Archanjo de Souza}

Doutoranda do Programa de Pós-Graduação em Engenharia Mecânica - Área de Projetos, Gestão e Otimização, UNESP - Campus de Guaratinguetá, 0000-0001-9089-1246, daniele.archanjo@hotmail.com

\section{Andreia Maria Pedro Salgado}

Professora Assistente Doutora do Programa de Pós-Graduação em Engenharia da Produção, UNESP / Campus de Guaratinguetá, 0000-0003-4733-4860, andreia.maria@unesp.br

\section{Édis Mafra Lapolli}

Doutora em Engenharia de Produção, Professora do Programa de Pós-graduação em Engenharia e Gestão do Conhecimento, UFSC,0000-0001-8534-7449, edispandion@gmail.com

\section{Fernando Augusto Silva Marins}

Professor Doutor do Programa de Pós-Graduação em Engenharia Mecânica, UNESP / Campus de Guaratinguetá, 0000-0001-6510-9187,fernando.marins@unesp.br

\section{RESUMO}

Objetivo: Mapear sistematicamente as produções científicas que tratam da relação entre a liderança e a capacidade absortiva do conhecimento. 
Design/Metodologia/Abordagem: Revisão bibliográfica sistemática, por meio da busca e análise das contribuições de pesquisas acadêmicas publicadas em artigos científicos que vinculam os construtos relacionados à liderança e capacidade absortiva do conhecimento.

Resultados: Na busca pelos temas na base de dados Scopus, foram encontradas 55 publicações e, após leitura dos resumos, foram selecionados 23 artigos que foram lidos e analisados por completo. Estes apresentam como destaque o ano de 2018 com o maior número de publicações; os autores García-Morales, Lloréns-Montes e Verdú-Jover (2008) com maior número de citações; o método quantitativo como o mais utilizado e o estilo de liderança transformacional como tema central.

Limitações da pesquisa: Artigos publicados na Plataforma Scopus até julho de 2019 e associados às palavras-chaves Liderança e Capacidade Absortiva.

Originalidade/valor: Não foram encontrados artigos que tratassem a relação dos temas na literatura de forma sistematizada. O diagnóstico dos problemas apresentados e a possibilidade de construir por meio de uma análise aprofundada, comparações e divergência entre as ideias, discussões e resultados apresentados pelos autores possibilitará o avanço da barreira do saber. Além disso, são identificadas oportunidades para novas pesquisas que são descritas em uma agenda de sugestões de trabalhos futuros, o que apoiará o desenvolvimento de novas pesquisas na área.

Palavras-chave: Liderança. Capacidade absortiva. Aprendizagem organizacional. Gestão do conhecimento. Revisão sistemática.

\title{
ANALYSIS OF THE RELATIONSHIP BETWEEN LEADERSHIP AND ABSORPTIVE KNOWLEDGE CAPACITY: A Systematic Literature Review
}

\begin{abstract}
Goal: Systematically map scientific productions that address the relationship between leadership and the absorptive capacity of knowledge.
\end{abstract}


Design / Methodology / Approach: Systematic literature review, through the search and analysis of the contributions of academic research published in scientific articles that link the constructs related to leadership and absorptive capacity of knowledge.

Results: In the search for the themes 55 articles were initially found and after reading the abstracts, 23 articles were selected which were thoroughly read and analyzed. 2018 as the year of greatest publication, García-Morales, Lloréns-Montes and Verdú-Jover (2008) as the most cited authors, the most widely used method as quantitative and as its central theme the transformational leadership style.

Limitations of the research (if applicable): Articles published on the Scopus Platform until July 2019 and associated with the keywords Leadership and Absorbing Capacity.

Originality / value: No articles were found that dealt with the relationship of themes in the literature in a systematic way. The diagnosis of the problems presented and the possibility of building through in-depth analysis, comparisons and divergence of the ideas, discussions and results presented by the authors will allow the advancement of the knowledge barrier. In addition, opportunities are identified for further research that are outlined in a suggested agenda for future work, which will support the development of new research in the area.

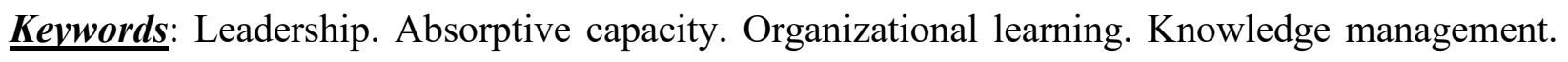
Systematic review 


\section{INTRODUÇÃO}

A constante busca de diferentes estratégias para que as organizações se mantenham competitivas diante da acirrada disputa de mercado na dinâmica atual dos negócios, torna fundamental a reformulação dos processos e o desenvolvimento de novas capacidades (Flatten, Adams, \& Brettel, 2015).

Nesse contexto, o estudo da Liderança e da sua relação com a Capacidade Absortiva de Conhecimento se justifica por pesquisas como as de Yaseen, Al-Janaydab, e Alc (2018), Rai e Prakash (2016), Kash, Spaulding, Gamm, e Johnson (2014) e García-Morales, Lloréns-Montes, e Verdú-Jover (2008), que descreveram que estes temas aparecem pouco vinculados e ainda relativamente inexplorados na literatura. Rai e Pakash (2016) ressaltam principalmente a carência de estudos empíricos que integrem influências moderadoras e mediadoras de diversos tipos na relação entre os construtos.

Desta forma, este artigo pretende identificar a relação entre a Liderança e a Capacidade Absortiva (CA) a partir do mapeamento sistemático da produção científica na base de dados Scopus. Com isso surge a questão de pesquisa: Qual a relação entre a liderança e a capacidade absortiva do conhecimento?

Como a liderança exerce um impacto direto na melhoria dos resultados (Avolio, Walumbwa, \& Weber, 2009; Vaccaro, Jansen, Van Den Bosch, \& Volberda, 2012) e por sua vez, a capacidade de absorção é de vital importância para a inovação e para intenções estratégicas de crescimento das empresas (Slavec Gomezel \& Rangus, 2019), autores como Jansen, Van Den Bosch, \& Volberda (2005), Rai e Prakash (2016), Supartha e Ratih (2017), Yaseen et al. (2018), Ferreras Méndez, Sanz Valle, e Alegre (2018) contribuíram ao verificar que a CA é afetada pela liderança e seus processos, validando, portanto, a existência da relação entre os temas em ambientes de Aprendizagem Organizacional (AO) e de Gestão do Conhecimento (GC).

Salienta-se que a pesquisa sobre os estilos de liderança, de forma específica, não será o foco do estudo, contudo tais estilos surgem de forma natural nos artigos analisados, à medida que os autores abordam a discussão sob diferentes perspectivas.

A estrutura deste artigo está organizada de acordo com a descrição a seguir: nas Seções 2 e 3 são apresentados o referencial teórico com os temas liderança, capacidade absortiva respectivamente; em seguida, na Seção 4 são descritos os procedimentos metodológicos adotados 
para desenvolver este estudo; na sequência, a Seção 5 descreve os principais resultados; e por fim, na Seção 6, estão as considerações finais, seguidas pelas referências utilizadas neste trabalho.

\section{LIDERANÇA}

A liderança, ao longo da evolução histórica, sempre apresentou um papel importante nas organizações nos âmbitos político, militar, social e industrial. Segundo Bass (2008), este fato ocorreu pelas constantes mudanças nos diferentes contextos e pelo aprofundamento das pesquisas no tema.

De maneira global, estudos conceituam Liderança como a influência sobre outro com o objetivo de alcançar metas (Vaitkevičius, 2016; Paliszkiewicz, Gołuchowski, \& Koohang, 2015; Birasnav, Rangnekar, \& Dalpati, 2011; Magnier-Watanabe, Benton, \& Senoo, 2011; Fujihara, 2010) por meio de esforço conjunto, visão e sucesso (Vaitkevičius, 2016; Donate \& Pablo, 2015).

Bergamini (1994) e Strapasson (2009) construíram esta evolução histórica das teorias sobre liderança representadas, inicialmente, pela Teoria do Grande Homem baseada na forte influência de grandes homens reconhecidos pela humanidade na história, e na evolução humana até as Teorias da Nova Liderança, que possuem como foco as pessoas, seu desenvolvimento, facilidade do líder para mudança, criatividade e capacidade de compartilhar uma visão futura. A representação principal desta última teoria, se refere aos estilos Transformacional e Transacional.

A Liderança Transformacional é definida como a capacidade de influência e de inspiração por meio de um perfil de líder que integra comportamentos de confiança, estímulo intelectual, motivação inspiradora e consideração individualizada Já a Liderança Transacional monitora o desempenho dos liderados e envolvem essencialmente transações por recompensa (Burns, 1978; Bass, 1985; Bass \& Avolio, 2004; Zhu, Avolio, Riggio, \& Sosik, 2011; Birasnav, 2014).

Diante do progresso na sociedade baseada no conhecimento, em que o comportamento do indivíduo se torna mais importante, parece apropriado que um líder assuma e desempenhe um papel que conduza sua equipe à realização de atividades baseadas no conhecimento (Lee, Lee, \& Park, 2014).

Este cenário requer que tais líderes sejam capazes de enfrentar uma realidade baseada no conhecimento e promover a inovação para alcançar melhorias no desempenho organizacional. No entanto, as organizações, por vezes, não conseguem obter vantagem competitiva sustentável 
devido a sua compreensão limitada das relações entre estas variáveis estratégicas (García-Morales et al., 2008).

Bass e Avolio (2004) e Yukl (2006) também contribuíram ao concluírem que a liderança, ao invés de simplesmente ser descrita como uma característica individual, é retratada em vários modelos como uma dinâmica social, compartilhada, relacional, estratégica, global e complexa. Neste sentido, Senge (1999) argumentou que a construção de uma visão compartilhada na organização é de extrema importância, uma vez que promove a necessidade de aprendizagem, exigindo o aprimoramento do conhecimento e a criação de novas capacidades dos líderes com o consequente direcionamento das atenções para o desenvolvimento da capacidade absortiva (Arbatani \& Mohammdpour, 2016).

\section{CAPACIDADE ABSORTIVA}

Cohen e Levinthal $(1989,1990)$ foram os primeiros a proporem um conceito para a capacidade absortiva do conhecimento, sendo, portanto, os autores mais citados quando se trata do tema. Partindo do princípio de que as empresas possuem capacidades de aprendizado diferenciadas, a CA de uma empresa é entendida pelos autores como um conjunto de habilidades da empresa para identificar, assimilar e aplicar conhecimento externo, com foco na inovação.

A CA apresenta forte ligação com a habilidade da organização de criar conhecimento (Cohen \& Levinthal, 1989), ou seja, gerar inovação; sendo também dependente do conhecimento prévio da empresa (Cohen \& Levinthal, 1990; Van Den Bosch, Volberda, \& De Boer, 1999; Zahra $\&$ George, 2002) e pode influenciar o desempenho organizacional e a vantagem competitiva da empresa (Zahra \& George, 2002).

Zahra e George (2002) propõem um novo conceito para a CA, que, segundo Todorova e Durisin (2007), não realiza avanços relevantes sobre as bases teóricas construídas por Cohen e Levinthal (1990). Não obstante, vários estudos têm recorrido às proposições desenvolvidas por Zahra e George (2002), principalmente no que se refere à subdivisão da capacidade absortiva em CA potencial e CA realizada. Os autores explicam que a CA potencial reflete a capacidade da empresa para adquirir e assimilar conhecimento externo e a CA realizada expressa a habilidade da firma de transformar e aproveitar o conhecimento assimilado.

Zahra e George (2002) também sugerem a existência de mecanismos de integração social que moderam a relação entre a capacidade potencial e realizada, facilitando o compartilhamento e 
a integração do conhecimento na empresa. Assim, gestores atentos ao desenvolvimento da CA devem preconizar a disposição de mecanismos de integração social por parte da organização.

A definição da CA proposta por Zahra e George (2002) é fundamentada sob a perspectiva das capacidades dinâmicas, em que a CA é reconhecida como uma capacidade dinâmica da organização que possibilita a aquisição, a assimilação, a transformação e o aproveitamento do conhecimento, para o aumento da vantagem competitiva. O termo "dinâmica" se refere aos ambientes com mudança tecnológica rápida e incertezas e, assim, as capacidades dinâmicas são habilidades e competências desenvolvidas pelas organizações nestes ambientes em constante evolução (Teece, Pisano, \& Shuen (1997). Os elementos da incerteza e mudanças tecnológicas rápidas também foram, previamente, abordados nos estudos de Cohen e Levinthal $(1989,1990)$.

Umas das propriedades da capacidade de absorção do conhecimento é a sua dependência do caminho decorrente dos efeitos da cumulatividade e da formação de expectativas para absorção de novos conhecimentos (Cohen \& Levinthal, 1990). Assim, o desenvolvimento da CA em uma área amplia a possibilidade de a organização absorver mais conhecimento na mesma área pela similaridade e permite o reconhecimento do valor de novos avanços tecnológicos que precisem ser adquiridos. Arbatani e Mohammdpour (2016) afirmam que, no momento em que a organização absorve, simula, internaliza e adquire conhecimento, ela cria capacidades dinâmicas que podem levar a processos de inovação.

Para Cohen e Levinthal (1990), o fluxo do conhecimento e a comunicação organizacional são elementos importantes para o desenvolvimento da $\mathrm{CA}$, tanto nas relações internas entre funcionários quanto na interação da empresa com o ambiente externo.

A partir de uma revisão sobre a capacidade de absorção do conhecimento, Lane, Koka, e Pathak (2006) defendem que a CA não deve ser dissociada de seu contexto e que a estratégia organizacional desempenha um papel fundamental no desenvolvimento da CA, carecendo de mais pesquisas sobre o tema, com vistas a gerar insights para os gestores. O estudo da interrelação da liderança e da CA como diretriz estratégica da organização se legitima, diante disto.

Todorova e Durisin (2007) introduziram o elemento "relações de poder" no modelo da CA que corresponde à influência de atores internos e externos à organização sobre os processos de absorção de conhecimento, mais especificamente os de valorização e aplicação do conhecimento. De acordo com os autores, as relações de poder internas podem facilitar ou dificultar os processos de alocação de recursos para o aproveitamento do novo conhecimento externo adquirido. O uso 
do poder é considerado componente intrínseco de uma liderança forte, que, conforme definição, influencia os outros a perseguirem determinado objetivo, dispondo da importante habilidade de ser sensitivo aos outros e entender as pessoas (Pfeffer, 1993).

\section{METODOLOGIA}

Esta pesquisa trata-se de uma pesquisa bibliográfica que, de acordo com Marconi e Lakatos (2005, p.158), "é um apanhado geral sobre os principais trabalhos já realizados, revestidos de importância, por serem capazes de fornecer dados atuais e relevantes relacionados com o tema".

O desenvolvimento deste artigo se dá pela revisão estruturada da literatura presente no produto científico existente, com o objetivo de realizar um mapeamento por meio da possibilidade de traçar uma trajetória sistemática das publicações com posterior análise aprofundada dos artigos relevantes que discutem os constructos (Santos, Maldonado, \& Santos, 2011).

\section{ETAPA 1 - BUSCA SISTEMÁTICA NA BASE DE DADOS}

Realizada na base Scopus, que abrange pesquisas em diferentes áreas com periódicos reconhecidos pela comunidade científica internacional, o mês de realização foi julho de 2019 e a estratégia de busca foi ("leadership" AND “absorptive capacity") com ocorrência no título, resumo e palavras-chave. Sem a restrição de filtros, o resultado foram 55 publicações das quais os dados foram exportados para o Software Excel, para gerenciamento e análise dos artigos.

A Figura 1 apresenta a quantidade de publicações por ano e demonstra que a quantidade tem oscilado nos últimos dez anos, entretanto, a tendência aponta um crescimento ao longo do tempo.

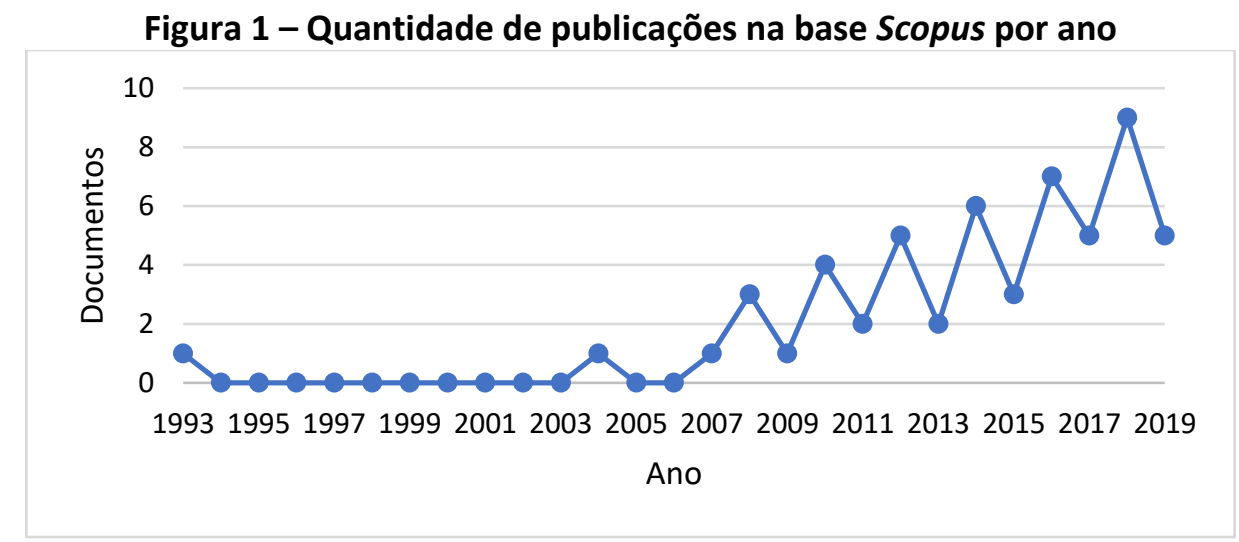

Fonte: Elaborado pelos autores 
Verificou-se que a maioria dos artigos são provenientes dos Estados Unidos (12) e do Reino Unido (11), não encontrando-se artigos de autoria brasileira, com os termos pesquisados, registrados na base neste período.

\section{ETAPA 2 - ANÁLISE SISTEMÁTICA DA LITERATURA}

Esta etapa consistiu na leitura de todos os títulos e resumos da etapa anterior que, de acordo com os critérios de relação específica com os temas propostos, foi possível identificar que alguns dos artigos não apresentavam a relação entre os constructos como seu principal conteúdo, e que alguns autores citaram o termo apenas para contextualizar o seu argumento primário e/ou para apontar implicações para futuras pesquisas.

A consequência foi a seleção de 23 artigos que foram lidos em sua totalidade e classificados no Quadro 1 de acordo com o número de citações na base e categorizados sob a perspectiva dos diferentes estilos de liderança-apresentados por cada autor.

\begin{tabular}{|c|c|c|c|c|}
\hline Autores & Ano & Títulos & $\begin{array}{l}\text { Estilo de } \\
\text { liderança } \\
\text { abordado }\end{array}$ & $\begin{array}{l}\mathrm{n} \text { o } \\
\text { cit. }\end{array}$ \\
\hline $\begin{array}{l}\text { García-Morales, } \\
\text { Lloréns-Montes e } \\
\text { Verdú-Jover }\end{array}$ & 2008 & $\begin{array}{c}\text { The effects of transformational leadership on } \\
\text { organizational performance through } \\
\text { knowledge and innovation }\end{array}$ & $\begin{array}{c}\text { Liderança } \\
\text { transformacional }\end{array}$ & 135 \\
\hline Sun & 2010 & $\begin{array}{l}\text { Five critical knowledge management } \\
\text { organizational themes }\end{array}$ & $\begin{array}{l}\text { Liderança } \\
\text { acolhedora }\end{array}$ & 44 \\
\hline $\begin{array}{c}\text { Flatten, Adams e } \\
\text { Brettel }\end{array}$ & 2015 & $\begin{array}{l}\text { Fostering absorptive capacity through } \\
\text { leadership: A cross-cultural analysis }\end{array}$ & $\begin{array}{l}\text { Liderança } \\
\text { transformacional } \\
\text { e liderança } \\
\text { transacional }\end{array}$ & 31 \\
\hline Sun e Anderson & 2012 & $\begin{array}{l}\text { The combined influence of top and middle } \\
\text { management leadership styles on absorptive } \\
\text { capacity }\end{array}$ & $\begin{array}{l}\text { Liderança } \\
\text { transformacional } \\
\text { e liderança } \\
\text { transacional } \\
\end{array}$ & 28 \\
\hline Lee, Lee e Park & 2014 & $\begin{array}{l}\text { Exploring the Impact of Empowering } \\
\text { Leadership on Knowledge Sharing, Absorptive } \\
\text { Capacity and Team Performance in it Service }\end{array}$ & $\begin{array}{c}\text { Liderança } \\
\text { empoderadora }\end{array}$ & 24 \\
\hline $\begin{array}{l}\text { McAdam, Reid e } \\
\text { Shevlin }\end{array}$ & 2014 & $\begin{array}{c}\text { Determinants for innovation implementation } \\
\text { at SME and inter SME levels within peripheral } \\
\text { regions }\end{array}$ & $\begin{array}{l}\text { Liderança para } \\
\text { inovação }\end{array}$ & 21 \\
\hline $\begin{array}{l}\text { Naqshbandi e } \\
\text { Tabche }\end{array}$ & 2018 & $\begin{array}{c}\text { The interplay of leadership, absorptive } \\
\text { capacity, and organizational learning culture in } \\
\text { open innovation: Testing a moderated } \\
\text { mediation model }\end{array}$ & $\begin{array}{c}\text { Liderança } \\
\text { empoderadora }\end{array}$ & 7 \\
\hline
\end{tabular}




\begin{tabular}{|c|c|c|c|c|}
\hline $\begin{array}{l}\text { Kash, Spaulding, } \\
\text { Gamm e Johnson }\end{array}$ & 2014 & $\begin{array}{l}\text { Leadership, culture, and organizational } \\
\text { technologies as absorptive capacity for } \\
\text { innovation and transformation in the } \\
\text { healthcare sector: A framework for research }\end{array}$ & $\begin{array}{l}\text { Liderança } \\
\text { convergente, } \\
\text { gerativa e } \\
\text { unificadora }\end{array}$ & 6 \\
\hline $\begin{array}{l}\text { Ferreras Méndez, } \\
\text { Sanz Valle e } \\
\text { Alegre }\end{array}$ & 2018 & $\begin{array}{c}\text { Transformational leadership and absorptive } \\
\text { capacity: an analysis of the organisational } \\
\text { catalysts for this relationship }\end{array}$ & $\begin{array}{c}\text { Liderança } \\
\text { transformacional }\end{array}$ & 5 \\
\hline $\begin{array}{l}\text { Daspit, } \\
\text { Ramachandran e } \\
\text { D'Souza }\end{array}$ & 2014 & $\begin{array}{l}\text { TMT shared leadership and firm performance: } \\
\text { Investigating the mediating role of absorptive } \\
\text { capacity }\end{array}$ & $\begin{array}{c}\text { Liderança } \\
\text { compartilhada }\end{array}$ & 5 \\
\hline Rai e Prakash & 2016 & $\begin{array}{c}\text { How do servant leaders ignite absorptive } \\
\text { capacity? the role of epistemic motivation and } \\
\text { organizational support }\end{array}$ & $\begin{array}{l}\text { Liderança } \\
\text { servidora }\end{array}$ & 3 \\
\hline $\begin{array}{l}\text { Matherly e Al } \\
\text { Nahyan }\end{array}$ & 2015 & $\begin{array}{l}\text { Workplace quotas: Building competitiveness } \\
\text { through effective governance of national- } \\
\text { expatriate knowledge transfer and } \\
\text { development of sustainable human capital }\end{array}$ & Liderança sênior & 3 \\
\hline $\begin{array}{l}\text { Chang, Chao, } \\
\text { Chang e Chi }\end{array}$ & 2018 & $\begin{array}{c}\text { Transformational leadership influence on unit } \\
\text { performance: Cross-level moderated } \\
\text { mediation evidence }\end{array}$ & $\begin{array}{c}\text { Liderança } \\
\text { transformaciona }\end{array}$ & 1 \\
\hline Supartha e Ratih & 2017 & $\begin{array}{l}\text { Antecedents of absorptive capacity: A proof of } \\
\text { proposition }\end{array}$ & Liderança criativa & 1 \\
\hline $\begin{array}{l}\text { Pérez-Aróstegui, } \\
\text { Sousa, Lloréns- } \\
\text { Montes }\end{array}$ & 2009 & $\begin{array}{l}\text { Quality management practices as a forerunner } \\
\text { of absorptive capacity. An empirical study }\end{array}$ & $\begin{array}{l}\text { Liderança como } \\
\text { prática de Gestão } \\
\text { da Qualidade } \\
\text { (GQ) }\end{array}$ & 1 \\
\hline $\begin{array}{c}\text { Chang, } \\
\text { Hodgkinson, } \\
\text { Hughes e Chang }\end{array}$ & 2019 & $\begin{array}{c}\text { The mediation between participative } \\
\text { leadership and employee exploratory } \\
\text { innovation: Examining intermediate } \\
\text { knowledge mechanisms }\end{array}$ & $\begin{array}{l}\text { Liderança } \\
\text { participativa }\end{array}$ & 0 \\
\hline $\begin{array}{l}\text { Slavec Gomezel e } \\
\text { Rangus }\end{array}$ & 2019 & $\begin{array}{l}\text { Open innovation: it starts with the leader's } \\
\text { openness }\end{array}$ & $\begin{array}{l}\text { Liderança aberta } \\
\text { e proativa }\end{array}$ & 0 \\
\hline Ramachandran & 2018 & $\begin{array}{c}\text { Triggering absorptive capacity in } \\
\text { organizations: CEO succession as a knowledge } \\
\text { enabler }\end{array}$ & $\begin{array}{l}\text { Liderança } \\
\text { estratégica }\end{array}$ & 0 \\
\hline $\begin{array}{l}\text { Wang, Zhao e } \\
\text { Zhou }\end{array}$ & 2018 & $\begin{array}{c}\text { How do incentives motivate absorptive } \\
\text { capacity development? The mediating role of } \\
\text { employee learning and relational } \\
\text { contingencies }\end{array}$ & $\begin{array}{c}\text { Liderança } \\
\text { transformacional }\end{array}$ & 0 \\
\hline $\begin{array}{c}\text { Yaseen, Al- } \\
\text { Janaydab e Alc }\end{array}$ & 2018 & $\begin{array}{l}\text { Leadership styles, absorptive capacity and } \\
\text { firm's innovation }\end{array}$ & $\begin{array}{c}\text { Liderança } \\
\text { transformacional } \\
\text { e liderança } \\
\text { transacional } \\
\end{array}$ & 0 \\
\hline $\begin{array}{l}\text { Darwish, Zeng, } \\
\text { Zadeh e Haak- } \\
\quad \text { Saheem }\end{array}$ & 2018 & $\begin{array}{c}\text { Organizational Learning of Absorptive } \\
\text { Capacity and Innovation: Does Leadership } \\
\text { Matter? }\end{array}$ & $\begin{array}{c}\text { Liderança } \\
\text { transformacional } \\
\text { e liderança } \\
\text { transacional }\end{array}$ & 0 \\
\hline
\end{tabular}




\begin{tabular}{|c|c|c|c|c|}
\hline Shafique e Beh & 2016 & $\begin{array}{c}\text { The role of leadership styles to promote } \\
\text { innovation: Empirical evidence from a } \\
\text { developing country }\end{array}$ & $\begin{array}{c}\text { Liderança } \\
\text { transformacional } \\
\text { e liderança } \\
\text { transacional }\end{array}$ & 0 \\
\hline $\begin{array}{c}\text { Arbatani e } \\
\text { Mohammadpour }\end{array}$ & 2016 & $\begin{array}{c}\text { Presenting a model for innovation excellence } \\
\text { in the islamic republic of iran tv }\end{array}$ & $\begin{array}{c}\text { Liderança em } \\
\text { inovação }\end{array}$ & 0 \\
\hline
\end{tabular}

Fonte: Elaborado pelos autores

\section{RESULTADOS E DISCUSSÕES}

Após a seleção dos artigos que comporiam a revisão sistemática da literatura, estes foram classificados e categorizados. O resultado fica caracterizado pelas seguintes métricas: 2018 como ano de maior publicação; a maior parte dos artigos apresenta o método quantitativo; os autores mais citados são García-Morales, Lloréns-Montes e Verdú-Jover (2008) e, como assunto central, tem-se o estilo de liderança transformacional.

O agrupamento dos estudos se deu pela indicação de comparações de aspectos considerados relevantes, sendo semelhantes, contraditórios ou complementares, identificados com base nas implicações da Liderança e da Capacidade Absortiva do conhecimento.

As perspectivas das análises individuais, registradas pelos autores, realizam um paralelo com temas complementares ao afirmar que a liderança exerce influência direta e significativa sobre a CA e por meio do Compartilhamento do Conhecimento (CC), resultando na melhoria da inovação e do desempenho organizacional (Daspit, Ramachandran, \& D’Souza, 2014; Lee, Lee \& Park, 2014; McAdam, Reid, \& Shevlin, 2014; Arbatani \& Mohammadpour, 2016; Supartha, \& Ratih, 2017; Chang, Chao, Chang, \& Chi, 2018; Chang, Hodgkinson, Hughes, \& Chang 2019; Slavec Gomezel \& Rangus, 2019). Não obstante, o estudo de Supartha e Ratih (2017) com Indústrias de Pequenos Artesãos não identificou relação direta da liderança com o aumento da CA, ressaltando a exigência da mediação do $\mathrm{CC}$.

Logo, a liderança é considerada um antecedente no caminho para a inovação e se relaciona positivamente com as dimensões mediadoras de aquisição e assimilação do conhecimento, representando a habilidade do líder em construir pontes e intermediar as redes de compartilhamento do conhecimento intra e interorganizacionais (McAdam, Reid, \& Shevlin, 2014). De modo complementar, a falta de CA pode determinar o sucesso da implementação de práticas de gestão como a liderança, havendo dessa maneira uma interação mútua entre os construtos (Pérez-Aróstegui, Sousa, \& Lloréns-Montes, 2009). 
Aspectos da liderança como o incentivo à motivação, a preocupação com as necessidades pessoais da equipe, a criação de novas oportunidades de aprendizagem associada às condições de apoio e a inspiração de esforços individuais, o estímulo ao desenvolvimento intelectual, à autoconfiança e à conscientização promovem a melhoria da capacidade da aprendizagem (Arbatani \& Mohammadpour, 2016). Neste sentido, Rai e Prakash (2016) propõem a influência da CA pela liderança servidora, pois esta desenvolve relações de cooperação, produzindo maior diálogo entre seus seguidores e disseminação de conhecimento.

Comportamentos do líder como liderar pelo exemplo, tomada de decisão participativa, coaching, informar e demonstrar preocupação consistem em elementos de empoderamento que proporcionam um aumento significativo na $\mathrm{CA}$ e os treinamentos para líderes devem abranger tais técnicas de empoderamento em situações da vida real (Lee, Lee \& Park, 2014). A liderança empoderadora é capaz de influenciar o comportamento dos funcionários da empresa, motivandoos a reproduzir o mesmo comportamento dos líderes em suas buscas por novos conhecimentos externos, favorecendo resultados inovadores por estimular a capacitação e a motivação dos funcionários para poderem tomar decisões independentes (Naqshbandi \& Tabche, 2018).

Ramachandran (2018) ressaltou a importância da liderança estratégica como facilitadora do conhecimento para a GC das organizações e sustentam, a partir de uma estrutura conceitual, que diferentes tipos de sucessão do CEO podem focar e dar preferência para diferentes aspectos do processo de GC. Assim, este autor entende a CA como uma capacidade dinâmica facilitadora da GC e a sucessão de CEO como um gatilho de ativação dos processos de GC envolvidos na CA.

Para Naqshbandi \& Tabche (2018) e Slavec Gomezel \& Rangus (2019), a liderança desempenha papel vital sobre a inovação aberta. Segundo Naqshbandi e Tabche (2018), a liderança empoderadora possibilita uma maior aquisição e fornecimento de conhecimento e um gerenciamento mais eficaz dos fluxos de saída de conhecimento promovendo a inovação aberta, entretanto, contrariando outros estudos, o papel moderador da CA na relação da cultura de aprendizagem com a inovação aberta, não foi confirmado por estes últimos autores. Já Slavec Gomezel e Rangus (2019) destacaram a importância do empreendedorismo individual do líder que predispõe especificamente a indivíduos com uma inclinação à novidade, à aprendizagem e à busca de feedback, o que impulsiona a CA das empresas.

Os líderes devem adotar um comportamento participativo, impulsionando o envolvimento dos funcionários na tomada de decisões, incentivando os debates nas reuniões e o uso de 
brainstorming, uma vez que isto promove oportunidades para o CC e estimula a inovação, por meio do aumento da capacidade absortiva dos funcionários, que conseguirão acessar informações que possibilitam assimilar e usar novos conhecimentos de forma autônoma e independente (Chang et al., 2019).

Para Sun (2010), o impacto da liderança sobre a GC depende de uma plataforma de rotinas e processos construídos para desenvolver as capacidades organizacionais, dentre elas a capacidade de absorção do conhecimento. Neste sentido, Matherly e Al Nahyan (2015) defenderam que os líderes devem criar ambientes favoráveis para promover a transferência de conhecimento, estabelecendo políticas que promovam uma cultura de transparência e confiança com incentivos baseados no alinhamento dos objetivos organizacionais. Segundo estes autores, para uma efetiva troca de conhecimento e desenvolvimento do capital humano, as pessoas envolvidas precisam se afastar de um modelo baseado em renda fundado no privilégio para um sistema que garanta a concorrência justa e igualdade de acesso às oportunidades.

Alguns estudos analisaram a influência de diferentes estilos de liderança sobre a CA, a saber, o transformacional e o transacional (Sun \& Anderson, 2012; Flatten et al., 2015; Shafique \& Beh, 2016; Darwish, Zeng, Rezaei Zadeh, \& Haak-Saheem, 2018; Yaseen, Al-Janaydab, \& Alc, 2018). Entretanto, apenas Sun e Anderson (2012), Flatten et al. (2015) e Yaseen, Al-Janaydab, e Alc (2018) identificaram relação positiva entre ambos estilos e a CA.

Shafique e Beh (2016) atestaram que a liderança transformacional afeta a inovação tanto diretamente quanto indiretamente através da capacidade de absorção, porém a mesma relação não é significante no caso da liderança transacional. Darwish et al. (2018) corroboraram com esta evidência, afirmando que não houve mediação na relação entre o processo interno de aprendizagem explorativa e a inovação. Flatten et al. (2015) contribuíram com esta afirmação, ao constatarem que a liderança transacional exerce efeito bem menor sobre a CA ao ser comparada com o estilo de liderança transformacional.

Kash et al. (2014), Chang et al. (2018), Ferreras Méndez, Sanz Valle, e Alegre (2018), descreveram em seus trabalhos que a eficácia da Liderança Transformacional depende do nível da CA organizacional. Já García-Morales, Lloréns-Montes e Verdú-Jover (2008) e Darwish et al. (2018) apresentaram o impacto direto desta relação com os processos de aprendizado e de inovação. 
Neste contexto, Ferreras Méndez, Sanz Valle e Alegre (2018) afirmaram que líderes transformacionais que são capazes de ganhar respeito e a confiança de seus seguidores, proporcionando-lhes uma missão inspiradora, apoio e encorajamento da sua criatividade, podem levar as empresas a obter níveis mais altos de CA, por estimularem a experimentação, a tomada de riscos, o diálogo e a interação com a comunidade externa.

De acordo com García-Morales, Lloréns-Montes e Verdú-Jover (2008) tais líderes devem estar ativamente envolvidos no desenvolvimento de pessoas, serem bons mentores, capazes de orientar os membros da equipe em suas trajetórias profissionais, apoiando o desenvolvimento de suas capacidades de aprendizagem e inovação.

Segundo Chang, Chao, Chang e Chi (2018), líderes transformacionais precisam melhorar a capacidade de absorção da empresa para adquirir, assimilar, transformar e explorar conhecimento para os fins comerciais. Para estes autores, treinamentos que geram estímulo para o CC nas equipes e práticas como job rotation foram identificadas como fatores de melhoria da CA, o que asseguraria o contínuo CC e a melhoria dos resultados.

Contrariamente à lógica convencional, Wang, Zhao, e Zhou (2018) verificaram uma relação negativa entre a liderança transformacional e os incentivos à inovação em sua pesquisa realizada com empresas automotivas da China. Os autores explicaram que questões culturais são a principal causa para este resultado, pois a consideração pessoal e o apoio dos líderes possuem grande importância para os chineses e qualquer percepção de favoritismo ou falta de transparência podem gerar desconfortos no ambiente de trabalho, dificultando assim os incentivos à inovação.

Para um melhor conhecimento das possíveis lacunas de pesquisa sobre o tema tratado neste estudo, uma agenda de sugestões de trabalhos futuros extraídas dos artigos analisados é apresentada no Quadro 2.

Quadro 2 - Sugestões de trabalhos futuros dos artigos selecionados na revisão sistemática

\begin{tabular}{|c|l|}
\hline Autores & \multicolumn{1}{c|}{ Sugestões de Trabalhos futuros } \\
\hline $\begin{array}{c}\text { Chang, } \\
\text { Hodgkinson, } \\
\text { Hughes e Chang } \\
(2019)\end{array}$ & $\begin{array}{l}\text { Necessidade de estudos mais amplos sobre CA em diferentes níveis. Integração da } \\
\text { liderança e da teoria de AO para explorar mais a dinâmica do conhecimento multinível } \\
\text { para avaliar a eficácia da liderança. }\end{array}$ \\
\hline $\begin{array}{c}\text { Slavec Gomezel } \\
\text { e Rangus (2019) }\end{array}$ & $\begin{array}{l}\text { Laços sociais fortes e fracos devem ser investigados em relação à CA, pois agregariam valor } \\
\text { na expansão do modelo e explicariam o papel dos empreendedores e de sua rede no } \\
\text { processo de inovação aberta. A relação com participação de mercado, ou crescimento de } \\
\text { receita, podem ser investigadas. }\end{array}$ \\
\hline
\end{tabular}




\begin{tabular}{|c|c|}
\hline $\begin{array}{l}\text { Chang, Chao, } \\
\text { Chang e Chi } \\
\text { (2018) }\end{array}$ & $\begin{array}{l}\text { O modelo pode ser expandido na tentativa de explorar a mediação da aquisição, recepção } \\
\text { ou integração de conhecimento. A pesquisa pode ser aplicada em diferentes níveis para } \\
\text { análise de deferentes resultados. }\end{array}$ \\
\hline $\begin{array}{l}\text { Darwish, Zeng, } \\
\text { Zadeh e Haak- } \\
\text { Saheem (2018) }\end{array}$ & $\begin{array}{l}\text { Estudo mais aprofundado dos processos, meios e mecanismos pelos quais diferentes } \\
\text { estilos de liderança podem ser transformados nos principais mecanismos que } \\
\text { impulsionam a capacidade de absorção. Aplicação do modelo em outros países com } \\
\text { contextos culturais e ramos de negócio diferentes. }\end{array}$ \\
\hline $\begin{array}{l}\text { Ferreras } \\
\text { Méndez, Sanz } \\
\text { Valle e Alegre } \\
\quad \text { (2018) }\end{array}$ & $\begin{array}{l}\text { As pesquisas poderiam ser realizadas além da percepção da alta gerência, o que poderia } \\
\text { fornecer uma avaliação mais completa sobre liderança transformacional. Incluir outros } \\
\text { estilos de liderança no modelo e estudar os diferentes processos incluídos na CA } \\
\text { separadamente. Examinar a combinação dos estilos transformacionais e transacionais e } \\
\text { seu impacto na promoção da CA. Também pode ser verificado como as condições do } \\
\text { ambiente afetam a relação entre liderança e conceito de CA. }\end{array}$ \\
\hline $\begin{array}{l}\text { Naqshbandi e } \\
\text { Tabche (2018) }\end{array}$ & $\begin{array}{l}\text { Pesquisas futuras podem testar o papel moderador da CA em diferentes contextos para } \\
\text { verificar se o contexto desempenha um papel, e também podem destacar outros estilos } \\
\text { de liderança que podem promover ou impedir a inovação aberta. Por fim, como utilizou- } \\
\text { se dados transversais para testar o modelo desenvolvido neste estudo, pesquisas futuras } \\
\text { podem testar o modelo usando dados longitudinais para determinar a causalidade com } \\
\text { maior confiança }\end{array}$ \\
\hline $\begin{array}{l}\text { Ramachandran } \\
\text { (2018) }\end{array}$ & $\begin{array}{l}\text { Analisar o impacto das características do CEO e da alta gerência como eles acionam os } \\
\text { componentes da CA como extensão dos estudos que analisaram a relação entre liderança } \\
\text { estratégica e CA. Realizar ajustes no framework e nas proposições do artigo considerando } \\
\text { o contexto dos negócios familiares. Investigar a influência de outros tipos de gatilhos de } \\
\text { ativação internos e externos sobre a CA. Testar empiricamente as proposições feitas pelo } \\
\text { artigo. }\end{array}$ \\
\hline $\begin{array}{l}\text { Wang, Zhao e } \\
\text { Zhou (2018) }\end{array}$ & $\begin{array}{l}\text { Realizar estudo longitudinal; ampliar pra outros tipos de empresas de manufatura e de } \\
\text { outros países. Validar de forma cruzada as análises usando dados de objeto de incentivos } \\
\text { à inovação. Considerar diferentes tipos de conhecimento (explícito, tácito, etc) e examinar } \\
\text { a interação de incentivos e outros mecanismos para motivar a transferência de } \\
\text { conhecimento tácito, por exemplo. }\end{array}$ \\
\hline $\begin{array}{l}\text { Yaseen, Al- } \\
\text { Janaydab e Alc } \\
\quad(2018)\end{array}$ & $\begin{array}{l}\text { Realizar estudo longitudinal; ampliar pra outros tipos de empresas intensivas em } \\
\text { conhecimento e de outros países. Comparar com outros tipos de empresas. Pesquisas } \\
\text { futuras poderiam examinar outras variáveis mediadoras, tais como, CA potencial, CA } \\
\text { realizada e compartilhamento do conhecimento. }\end{array}$ \\
\hline $\begin{array}{l}\text { Supartha e Ratih } \\
\text { (2017) }\end{array}$ & $\begin{array}{l}\text { Este estudo só pode dar uma visão geral sobre o caráter da capacidade de absorção no } \\
\text { ano atual e, portanto, a pesquisa longitudinal precisa ser conduzida. }\end{array}$ \\
\hline $\begin{array}{c}\text { Arbatani e } \\
\text { Mohammadpour } \\
(2016)\end{array}$ & $\begin{array}{l}\text { Investigar o método em outras organizações. Utilização de métodos qualitativos e mistos } \\
\text { para identificar fatores efetivos nos resultados. }\end{array}$ \\
\hline $\begin{array}{l}\text { Rai e Prakash } \\
\text { (2016) }\end{array}$ & $\begin{array}{l}\text { Realizar estudos longitudinais; capturar o impacto diferencial da natureza e do tipo de } \\
\text { organizações no comportamento do conhecimento. Estudo do preconceito dentro de um } \\
\text { contexto cultural. Realizar trabalhos que analisem outros aspectos do comportamento } \\
\text { individual da aprendizagem como a ocultação de conhecimento, conhecimento com } \\
\text { retenção e armazenamento de conhecimento para alcançar uma compreensão sistêmica } \\
\text { do tema. }\end{array}$ \\
\hline
\end{tabular}




\begin{tabular}{|c|c|}
\hline $\begin{array}{l}\text { Shafique e Beh } \\
\text { (2016) }\end{array}$ & $\begin{array}{l}\text { Estudos futuros poderiam examinar os efeitos de cada dimensão da liderança } \\
\text { transformacional na inovação. Da mesma forma, outros estudos também podem avaliar a } \\
\text { mediação de cada dimensão da CA. }\end{array}$ \\
\hline $\begin{array}{c}\text { Flatten, Adams e } \\
\text { Brettel (2015) }\end{array}$ & $\begin{array}{l}\text { Analisar o impacto das duas dimensões dos dois estilos de liderança (transformacional e } \\
\text { transacional) ou avaliar a relação entre os dois comportamentos de liderança e CA, a fim } \\
\text { de confirmar que os melhores líderes. }\end{array}$ \\
\hline $\begin{array}{l}\text { Matherly e Al } \\
\text { Nahyan (2015) }\end{array}$ & $\begin{array}{l}\text { Desenvolvimento de uma agenda integrada de pesquisas futuras sobre as áreas de gestão } \\
\text { estratégica, teoria de agências, GC e CA. }\end{array}$ \\
\hline $\begin{array}{l}\text { Daspit, } \\
\text { Ramachandran e } \\
\text { D'Souza (2014) }\end{array}$ & $\begin{array}{l}\text { Examinar a capacidade de absorção com o aumento do tamanho da amostra em uma } \\
\text { gama mais ampla de empresas de diferentes setores da indústria. Replicar e ampliar o } \\
\text { estudo atual examinando os fenômenos ao longo do tempo, obtendo provavelmente uma } \\
\text { melhor compreensão do comportamento de liderança, das influências de longo prazo na } \\
\text { CA e dos efeitos no desempenho. }\end{array}$ \\
\hline $\begin{array}{l}\text { Kash, Spaulding, } \\
\text { Gamm e } \\
\text { Johnson (2014) }\end{array}$ & $\begin{array}{l}\text { Capturar e rastrear perfis de liderança, cultura e Tecnologias Organizacionais ajudará } \\
\text { pesquisadores e líderes a desenvolverem estratégias que melhorem a implementação e o } \\
\text { sucesso da transformação. Tomar decisões informadas sobre o momento e a seleção de } \\
\text { iniciativas e decidir se devem ou não buscar ou expandir transformações específicas } \\
\text { dentro de programas, equipes ou sistemas inteiros. }\end{array}$ \\
\hline $\begin{array}{l}\text { Lee, Lee e Park } \\
\text { (2014) }\end{array}$ & $\begin{array}{l}\text { Medir e monitorar o processo de capacitar liderança e flutuações no compartilhamento } \\
\text { de conhecimento e capacidade de absorção em Projetos de Tecnologia da Informação (TI). }\end{array}$ \\
\hline $\begin{array}{l}\text { McAdam, Reid e } \\
\text { Shevlin (2014) }\end{array}$ & $\begin{array}{l}\text { Comparar os resultados com pequenas e médias empresas de outras regiões. Ampliar o } \\
\text { número de estudos de caso (estudo realizado em apenas uma empresa) ou realizar } \\
\text { pesquisas empíricas qualitativas sobre o tema. }\end{array}$ \\
\hline $\begin{array}{c}\text { Sun e Anderson } \\
(2012)\end{array}$ & $\begin{array}{l}\text { Realizar estudos empíricos quantitativos a partir de coleta de dados de várias empresas. } \\
\text { Verificar como os comportamentos do líder seriam percebidos pelos indivíduos e como } \\
\text { isso poderia afetar os processos de aprendizagem da CA. Realizar estudos longitudinais } \\
\text { para determinar melhor se o sucesso de certas fases dos processos de aprendizagem da } \\
\text { CA são definidos por certas combinações de estilo de liderança de alta e média gerência, } \\
\text { considerando certas características do contexto organizacional. }\end{array}$ \\
\hline Sun (2010) & $\begin{array}{l}\text { Testar as proposições: O contexto organizacional de conhecimento sistêmico, } \\
\text { engajamento estratégico, redes sociais (externas e internas), contexto cultural e contexto } \\
\text { de processo e estrutural influenciam na eficácia do conhecimento na organização } \\
\text { (aquisição, criação e compartilhamento)? Realizar outros estudos empíricos. }\end{array}$ \\
\hline $\begin{array}{l}\text { Pérez-Aróstegui, } \\
\text { Sousa e Lloréns- } \\
\text { Montes (2009) }\end{array}$ & $\begin{array}{l}\text { A análise da influência da implementação de práticas de GQ em outros processos de GC, } \\
\text { devido à escassez de estudos empíricos no campo. A determinação do efeito dos } \\
\text { antecedentes propostos nas diferentes dimensões da CA }\end{array}$ \\
\hline $\begin{array}{l}\text { García-Morales, } \\
\text { Lloréns-Montes } \\
\text { e Verdú-Jover } \\
\text { (2008) }\end{array}$ & $\begin{array}{l}\text { Fatores como estratégia, visão compartilhada, trabalho em equipe e tecnologia podem ser } \\
\text { abordados. A pesquisa também deve discutir melhoria da qualidade e melhorias na } \\
\text { capacidade relacional. Dedicar mais atenção à mensuração do desempenho dos negócios } \\
\text { a partir de uma perspectiva multidimensional. O desenvolvimento de um esquema } \\
\text { colaborativo poderia gerar uma estratégia organizacional em torno do conceito de } \\
\text { "liderança transformacional", para representar uma vantagem competitiva sustentável. } \\
\text { Estudos futuros devem ser baseados em amostras maiores, de preferência em um país. }\end{array}$ \\
\hline
\end{tabular}

Fonte: Elaborado pelos autores

Conforme Quadro 2, a maioria dos estudos possuem limitações referentes ao local de aplicação da pesquisa (tipo de empresa, país) e recomendam que a mesma seja expandida para 
outras localidades e contexto organizacional. Os autores também sugerem que, para analisar o fenômeno ao longo do tempo, estudos longitudinais sejam realizados para avaliar as diversas relações entre liderança, CA, GC, inovação, desempenho, dentre outros.

\section{CONSIDERAÇÕES FINAIS}

Ao realizar a pesquisa com a análise sistemática da literatura sobre a liderança e a capacidade absortiva, este trabalho disponibiliza um mapeamento da produção científica internacional sobre os temas e agrega informações úteis para a desenvolvimento de novos estudos e publicações sobre o assunto.

Não foram observadas a existência de outros artigos estruturados como revisão sistemática da literatura que tratassem da relação entre a CA e a liderança, comprovando-se, assim, a originalidade do artigo.

Os resultados da análise dos artigos mostraram que há uma interação mútua entre os construtos da capacidade absortiva e liderança. Contudo, a influência da liderança sobre a CA tem maior relevância pela quantidade de estudos.

É importante ressaltar que a pesquisa sobre os estilos de liderança não foi o propósito dessa pesquisa, contudo à medida que os artigos foram analisados, esse resultado surgiu de forma natural em função das diferentes perspectivas abordadas pelos diversos autores, o que permite concluir que o estilo de liderança transformacional recebeu maior destaque nos trabalhos analisados e a sua relação positiva com a CA foi comprovada nos estudos.-Porém, um deles observou que a cultura pode influenciar a relação entre a liderança transformacional, os incentivos à inovação e, por consequência, a CA, podendo gerar um impacto negativo.

Futuros estudos podem ampliar a pesquisa em diferentes bases, tanto internacionais como nacionais, por meio da realização de comparações das combinações dos diferentes achados que foram compilados neste trabalho. Além disso o cruzamento com outras palavras-chaves pode ser incluído para uma amostra mais específica e para verificação do impacto das etapas da CA, das fases da GC e dos diferentes estilos de liderança contidos na literatura atual.

Em suma, os resultados apresentados neste trabalho confirmaram a relação existente entre os constructos e o potencial de exploração das informações disponibilizadas podem ser utilizadas para nortear pesquisadores que forem realizar seus estudos no assunto exposto. 


\section{REFERÊNCIAS}

Arbatani, T. R., \& Mohammadpour, A. (2016). Presenting a model for innovation excellence in the Islamic Republic of Iran TV. International Journal of Applied Business and Economic Research, 14(3), 1851-1864.

Avolio, B. J., Walumbwa, F. O., \& Weber, T. J. (2009). Leadership: Current Theories, Research, and Future Directions. Annual Review of Psychology, 60, 421-449.

Bass, B. (1985). Leadership: Good, better, best. Organizational Dynamics, 13(3), 26-40.

Bass, B., \& Avolio, B. (2004). Multifactor Leadership questionnaire: Manual and Sampler. California, Mind Garden: Academic Press.

Bass, B. M., \& Bass, R. (2008). Handbook of Leadership: Theory, research, and application $\left(1^{\mathrm{a}}\right.$ ed). New York: Free Press.

Bergamini, C. (1994). Liderança: a administração do sentido. RAE - Revista de Administração de Empresas, 34(3), 102-114.

Birasnav, M. (2014). Knowledge management and organizational performance in the service industry: The role of transformational leadership beyond the effects of transactional leadership, Journal of Business Research, 67(8), 1622-1629.

Birasnav, M., Rangnekar, S., \& Dalpati, A. (2011). Transformational leadership and human capital benefits: The role of knowledge management. Leadership \& Organization Development Journal, 32(2), 106-126.

Burns, J. (1978). Leadership. New York: Perenium.

Chang, Y. Y., Chao, W. C., Chang, C. Y., \& Chi, H. R. (2018). Transformational leadership influence on unit performance: Cross-level moderated mediation evidence. Leadership \& Organization Development Journal, 39(4), 554-571.

Chang, Y. Y., Hodgkinson, I., Hughes, P., \& Chang, C. Y. (2019). The mediation between participative leadership and employee exploratory innovation: Examining intermediate knowledge mechanisms. Leadership \& Organization Development Journal, 40(3), 334-355.

Cohen, W. M., \& Levinthal, D. A. (1989). Innovation and learning: The two faces of R\&D. The Economic Journal, 99(397), 569-596.

Cohen, W. M., \& Levinthal, D. A. (1990). Absorptive capacity: A new perspective on learning and innovation. Administrative Science Quarterly, 35(1), 128-152.

Darwish, T. K., Zeng, J., Rezaei Zadeh, M., \& Haak-Saheem, W. (2018). Organizational Learning of Absorptive Capacity and Innovation: Does Leadership Matter? European Management Review. 
Daspit, J. J., Ramachandran, I., \& D’Souza, D. E. (2014). TMT shared leadership and firm performance: Investigating the mediating role of absorptive capacity. Journal of Managerial Issues, 26(3), 219-239.

Donate, M. J. \& de Pablo, J. D. S. (2015). The role of knowledge-oriented leadership in knowledge management practices and innovation. Journal of Business Research, 68 (2), 360-370.

Ferreras Méndez, J. L., Sanz Valle, R., \& Alegre, J. (2018). Transformational leadership and absorptive capacity: an analysis of the organisational catalysts for this relationship. Technology Analysis \& Strategic Management, 30(2), 211-226.

Flatten, T., Adams, D., \& Brettel, M. (2015). Fostering absorptive capacity through leadership: A cross-cultural analysis. Journal of World Business, 50(3), 519-534.

Fujihara, R. K. (2010). Gestão do conhecimento estratégico: estudo dos subfatores sistêmicos aplicados à ECT. Dissertação (Mestrado em Ciência da Informação) - Universidade de Brasília, Brasília.

García-Morales, V. J., Lloréns-Montes, F. J., \& Verdú-Jover, A. J. (2008). The effects of transformational leadership on organizational performance through knowledge and innovation. British Journal of Management, 19(4), 299-319.

Jansen, J. J., Van Den Bosch, F. A., \& Volberda, H. W. (2005). Managing potential and realized absorptive capacity: how do organizational antecedents matter?. Academy of management journal, 48(6), 999-1015.

Kash, B. A., Spaulding, A., Gamm, L. D., \& Johnson, C. (2014). Leadership, culture, and organizational technologies as absorptive capacity for innovation and transformation in the healthcare sector: A framework for research. Change Management, 13(1), 1-13.

Lane, P. J., Koka, B. R., \& Pathak, S. (2006). The reification of absorptive capacity: A critical review and rejuvenation of the construct. Academy of management review, 31(4), 833-863.

Lee, J., Lee, H., \& Park, J. G. (2014). Exploring the impact of empowering leadership on knowledge sharing, absorptive capacity and team performance in IT service. Information Technology \& People, 27(3), 366-386.

Magnier-Watanabe, R., Benton, C., \& Senoo, D. (2011). A study of knowledge management enablers across countries Knowledge Management Research \& Practice. Operational Research Society, 9(1), 17-28.

Marconi, M. De A., \& Lakatos, E. M. (2005). Fundamentos de metodologia científica. 6. ed. São Paulo: Atlas.

Matherly, L. L., \& Al Nahyan, S. S. (2015). Workplace quotas: Building competitiveness through effective governance of national-expatriate knowledge transfer and development of sustainable human capital. International Journal of Organizational Analysis, 23(3), 456-471. 
McAdam, R., Reid, R., \& Shevlin M. (2014). Determinants for innovation implementation at SME and inter SME levels within peripheral regions. International Journal of Entrepreneurial Behaviour \& Research, 20(1), 66-90.

Naqshbandi M. M., \& Tabche I. (2018). The interplay of leadership, absorptive capacity, and organizational learning culture in open innovation: Testing a moderated mediation model. Technological Forecasting and Social Change, 133, 156-167.

Paliszkiewicz, J., Gołuchowski, J., \& Koohang, A. (2015). Leadership, trust, and knowledge management in relation to organizational performance: Developing an instrument. The Online Journal of Applied Knowledge Management, 3(2), 19-35.

Petticrew, M., \& Roberts, H. (2006). Systematic reviews in the social sciences: a practical guide. 2006. Malden USA: Blackwell Publishing.

Pérez-Aróstegui, M. N., Sousa, R., \& Lloréns-Montes, J. (2009). Quality management practices as a forerunner of absorptive capacity: An empirical study. Investment Management and Financial Innovations, 6(3), 264-272.

Pfeffer, J. (1992). Managing with power: Politics and influence in organizations. Harvard Business Press.

Rai, R., \& Prakash, A. (2016). How do servant leaders ignite absorptive capacity? the role of epistemic motivation and organizational support. Journal of Work and Organizational Psychology, 32(2), 123-134.

Ramachandran, I. (2018). Triggering absorptive capacity in organizations: CEO succession as a knowledge enabler. Journal of Knowledge Management, 22(8), 1844-1864.

Santos, J. L. S., Maldonado, M. U., \& Santos, R. N. M. (2011). Inovação e conhecimento organizacional: um mapeamento bibliométrico das publicações científicas até 2009. Organizações em Contexto, 7(13), 31-58.

Senge, P. (1999). It's the learning: The real lesson of the quality movement. The Journal for Quality and Participation, 22(6), 34.

Shafique I., \& Beh L.-S. (2016). The role of leadership styles to promote innovation: Empirical evidence from a developing country. International Journal of Business Research, 16(1), 23-38. Slavec Gomezel, A., \& Rangus, K. (2019). Open innovation: it starts with the leader's openness. Innovation: Organization and Management, 1-19.

Strapasson, M. (2009). Liderança Transformacional na Enfermagem. Revista Brasileira de Enfermagem, Lajeado, 62(2), 228-233. 
Sun, P. (2010). Five critical knowledge management organizational themes. Journal of Knowledge Management, 14(4), 507-523.

Sun, P. Y. T., \& Anderson, M. H. (2012). The combined influence of top and middle management leadership styles on absorptive capacity. Management Learning, 43(1), 1-27.

Supartha W. G., \& Ratih I. A. D. K. (2017). Antecedents of absorptive capacity: A proof of proposition. Journal of Business and Retail Management Research, 11(4), 90-107.

Teece, D. J., Pisano, G., \& Shuen, A. (1997). Dynamic capabilities and strategic management. Strategic management journal, 18(7), 509-533.

Todorova, G., \& Durisin, B. (2007). Absorptive capacity: Valuing a reconceptualization. Academy of management review, 32(3), 774-786.

Vaccaro, I. G., Jansen, J. J., Van Den Bosch, F. A., \& Volberda, H. W. (2012). Management innovation and leadership: The moderating role of organizational size. Journal of Management Studies, 49(1), 28-51.

Vaitkevičius, V. (2016). Lyderystès vaidmuo formuojant žinių valdymui palankią organizacijos kultūrą: atvejo analizè. Informacijos mokslai, 76, 123-138.

Van Den Bosch, F. A. J., Volberda, H. W., \& De Boer, M. (1999). Coevolution of firm absorptive capacity and knowledge environment: Organizational forms and combinative capabilities. Organization science, 10(5), 551-568.

Wang L., Zhao J. Z., \& Zhou K. Z. (2018). How do incentives motivate absorptive capacity development? The mediating role of employee learning and relational contingencies. Journal of Business Research, 85, 226-237.

Yaseen, S. G., Al-Janaydab, S., \& Alc, N. A. (2018). Leadership styles, absorptive capacity and firm's innovation. International Journal of Knowledge Management, 14(3), 82-100.

Yukl, G. (1989). Managerial Leadership: A Review of Theory and Research. Journal of Management, 15(2), 83-86.

Zahra, S. A., \& George, G. (2002). Absorptive capacity: A review, reconceptualization, and extension. Academy of management review, 27(2), 185-203.

Zhu, W., Avolio, B., Riggio, R., \& Sosik, J. (2011). The effect of authentic transformational leadership on follower and group ethics. Leadership Quarterly, 22(5), 801-817. 